\title{
Critério para Implantação de Tecnologias de Suprimentos de Água Potável em Municípios Cearenses Afetados pelo Alto Teor de Sal
}

\author{
Valéria Pereira Monteiro ${ }^{1}$ \\ José César Vieira Pinheiro ${ }^{2}$
}

Resumo - O presente artigo tem por objetivo apresentar um critério para implantação de tecnologias de suprimento de água potável em municípios cearenses afetados pelo alto teor de sal nos mananciais que abastecem as suas populações . Partindo-se da hipótese, comprovada no trabalho, de que existe forte correlação entre as condições socioeconômicas e o teor de sal da água que abastece a população, elegeu-se o critério socioeconômico. Por intermédio da técnica de análise fatorial pelo método dos componentes principais e a partir dos indicadores socioeconômicos e ambientais de cada município, foram construídos índices a partir dos quais se efetuou uma hierarquização dos municípios estudados.

Palavras-chaves: critério, socioeconômico, hierarquização , municípios, cearense.

Abstract - The present article intend to present a criterion for implantation of technologies destined to desalinated the drinking waters reser-

\footnotetext{
${ }^{1}$ Mestre em Economia Rural pela Universidade Federal do Ceará e doutorando do programa de Pós Graduação em Economia Rural da Universidade Federal do Ceará.

E-mail: pedroevaleria@uol.com.br

${ }^{2}$ Prof. Doutor do Departamento de Economia Agrícola-CCA/UFC e pesquisador do CNPq. E-mail: jcvpinhe@ufc.br
} 
voirs that provide the cities of the state of Ceara affected by high levels of salt. Considering the hypothesis, demonstrated in this article, that there is a strong correlation between the socioeconomics conditions and the level of salt of the water that supplies the population of the cities, a socioeconomic criterion was chosen. Applying the technique of factorial analysis for the method of the main components to analyze socioeconomic and environmental variables, it could be arrange the cities in hierarchy order according to its needs of receive desalination equipment.

Key-Word - criterion, socioeconomic, hierarchization, factorial analysis, Ceará.

Jel Classification: Q25 e Q16

\section{1 - Introdução}

A escassez de água potável que desponta nesse novo milênio como uma das principais preocupações da humanidade, há muito faz parte da realidade do Nordeste brasileiro. Vastas áreas do semi-árido nordestino sofrem com o problema crônico das baixas precipitações pluviométricas e das secas periódicas, somados à ocorrência freqüente de elevados níveis de salinização dos seus mananciais.

A salinização das águas no Nordeste brasileiro vem se revelando como um fenômeno tão preocupante quanto a baixa precipitação. Para se ter uma idéia da dimensão do problema, cumpre registrar que por meio do censo realizado em 1997/2000 pela Companhia de Pesquisas de Recursos Minerais (CPRM), núcleo Fortaleza, constatou-se que, no Ceará, das 13.970 fontes de água subterrânea cadastradas e caracterizadas, 3.256 possuem teor de sal acima de $1000 \mathrm{mg} / \mathrm{litro}$ de sólidos totais dissolvidos $^{3}$, com a estimativa de que esses poços são fonte de abastecimento de água para consumo de cerca de 200.000 famílias (CPRM- 2000).

\footnotetext{
${ }^{3}$ São níveis que extrapolam o aceitável para o consumo humano. A Organização Mundial da Saúde (OMS) considera essa (1000mg/litro de STD) a quantidade máxima admitida na água, acima da qual a água perde sua potabilidade e sua ingestão poderá produzir danos à saúde humana.
} 
Assim, esse fenômeno vem se tornando objeto de importantes estudos que, dentre outras coisas, procuram explicar suas causas, desenvolver técnicas de medição do teor de sal nas águas e desenvolver tecnologias de dessalinização (REBOUÇAS, 1973; MATSUI, 1978; SANTIAGO, 1984; BARROS, 1994 e CEARÁ,1996). Há, também, aqueles que buscam apontar a melhor solução para o problema. É o caso dos estudos de SRH,1996 e Flores, 2000. Estes indicam a instalação de equipamentos de dessalinização como a alternativa técnica e economicamente mais adequada.

Apesar do mercado já dispor de tecnologia de dessalinização a custos cada vez mais reduzidos, estes ainda representam um gasto muito acima da capacidade financeira dos municípios cearenses afetados pelo fenômeno da salinização. Na maioria dos casos, faz-se necessário, portanto, um suporte financeiro das outras esferas de governo, seja estadual, seja federal, para viabilizar a instalação dessa infra-estrutura de dessalinização da água.

Nessas circunstâncias, a implantação desses equipamentos como forma de solução do problema de escassez de água potável nas diversas localidades do Estado do Ceará deverá vir inserida num projeto maior dos governos federal e/ou estadual. Os recursos, também, nessas esferas de governo, no entanto, não são ilimitados e devem ser alocados de forma racional e criteriosa. Portanto, para que se potencialize os benefícios gerados pela aplicação destes recursos, isto é, para que alcance a parcela da população mais severamente afetada, faz-se necessário um estudo que indique, a partir de critérios objetivos, quais municípios deverão receber, prioritariamente, essa ação governamental.

Neste sentido, o presente artigo se propõe efetuar uma hierarquização dos municípios cearenses afetados pela incidência de alto teor de sais de suas águas, a qual poderá servir de base para a definição de uma escala de prioridade para a instalação de equipamentos de dessalinização (ou de qualquer outra tecnologia que se revele mais adequada para solução do problema).

O critério que vem sendo preferencialmente adotado nesta priorização é o grau de salinização dos poços de cada município. Uma variação desse critério seria associar o grau de salinização à vazão dos poços. Para essas duas opções, basta o levantamento geológico com 
indicativo da vazão dos poços e do grau de salinidade de suas águas. A partir destes dados, se efetuaria a hierarquização dos municípios. Essas opções foram, neste trabalho, afastadas, pois se acredita que poderiam produzir resultados distorcidos. Por exemplo, determinado município, embora apresentando elevados níveis de salinização das águas de seu subsolo, poderia dispor de fonte alternativa de abastecimento em condições favoráveis. Pelo critério ora referido, esse município deveria ser beneficiado com instalação dos equipamentos, preterindo outro que, embora com níveis de salinização menores, não dispõe de boas fontes alternativas.

Nota-se, portanto, que um bom critério deverá agregar, pelo menos, três indicadores: teor de sal, vazão do poço e informações a respeito de fontes alternativas. As informações disponíveis sobre este último, todavia, não se apresentam de forma satisfatória. Elas têm revelado apenas existência ou não de fontes alternativas e a sua distância do poço. Outras, igualmente importantes, tais como a qualidade da água, a quantidade disponível e a freqüência ao longo do ano, ainda não estão disponíveis.

Na tentativa de contornar essas limitações metodológicas e de informações, este trabalho propõe um critério alternativo que busca efetuar a hierarquização dos municípios a partir de um conjunto de informações socioeconômicas e ambientais, inclusive as referentes ao grau de salinização das águas consumidas pelas populações desses municípios. Parte-se da hipótese de que a qualidade da água, aqui definida pelo alto teor de sal, tem forte correlação com as condições socioeconômicas das populações estudadas.

Com isso, espera-se oferecer um instrumento capaz de melhor orientar o uso de tecnologias voltadas para o abastecimento de água potável e, portanto, proporcionar uma melhor alocação dos recursos públicos.

Feitas estas considerações, cumpre afirmar que o presente trabalho tem como objetivo principal apresentar um critério para definição de uma escala de prioridades para instalação de tecnologias capazes de enfrentar o problema de abastecimento de água potável nos municípios cearenses que apresentam poços com altos teores de salinização das águas. Especificamente, espera-se: i) construir um índice para auxiliar 
o processo de tomada de decisão na definição de prioridades na instalação de tecnologias de suprimento de água potável em municípios do Estado do Ceará; ii) mostrar a influência do grau de salinização da água para o consumo humano nos indicadores socioeconômicos desses municípios, e iii) demonstrar um critério mais preciso para definição de prioridades na alocação de tecnologia de suprimento de água potável nesses municípios cearenses frente aos critérios existentes.

\section{2-Metodologia}

\subsection{Seleção das Variáveis Socioeconômicas e Ambientais}

O estudo se restringe ao Estado do Ceará, que tem a maior parte de seu território encravado no semi-árido. Serão analisados dados de todos os municípios que apresentarem pelo menos um poço com sólidos totais dissolvidos-STD- acima de $1000 \mathrm{mg} /$ litro.

Numa primeira fase, efetua-se a análise das estatísticas disponíveis na Fundação Instituto Brasileiro de Geografia e Estatística (IBGE), Fundação Instituto de Pesquisa e Informação do Ceará (IPLANCE) e Companhia de Pesquisa de Recursos Minerais (CPRM), de onde serão definidas e selecionadas todas as variáveis socioeconômicas e ambientais ${ }^{4}$, referentes a cada um dos 170 municípios estudados, conforme descrição a seguir:

$\mathrm{X}_{1}$, taxa de escolarização do ensino fundamental (1998) - indica a porcentagem da população de 7 a 14 anos escolarizada em relação à população escolarizável;

\footnotetext{
${ }^{4}$ Por limitação do programa de computador utilizado para efetuar a análise fatorial, as variáveis envolvidas no modelo deverão, todas, influenciar o resultado positivamente ou, todas, negativamente, não sendo possível que umas influenciem positivamente e outras negativamente. Quando isto não ocorre, faz-se necessário utilizar certos artifícios que as tornam homogêneas; isto é, utiliza-se o recurso de adotar o complementar, o inverso, etc. Por exemplo, a variável taxa de evasão escolar. Trata-se de uma variável que tem relação inversa à condição socioeconômica, porém o seu complementar, que seria taxa de permanência, estabelece relação positiva. Daí a utilização do seu complementar. Pelo mesmo motivo é que se usou o inverso para as variáveis: mortalidade infantil, salinidade média da água e outras.
} 
$\mathrm{X}_{2}$ - percentual de alunos aprovados no ensino fundamental (1998) indica o percentual dos alunos matriculados que foram promovidos para a série subseqüente;

$\mathrm{X}_{3}$ - complementar de alunos evadidos no ensino fundamental (1998) taxa de evasão indica a porcentagem de alunos matriculados na série $X$, no início do ano, que no ano seguinte não constam da matrícula, nem como alunos promovidos nem como alunos repetentes;

$\mathrm{X}_{4}$ - número de médicos por 1000 habitantes (1998) - mostra o número de médicos para um contingente de 1000 habitantes;

$\mathrm{X}_{5}$ - número de dentistas por 1000 habitantes (1998) - mostra o número de dentistas para um contingente de 1000 habitantes;

$\mathrm{X}_{6}$ - número de empresas industriais ativas por 1000 habitantes (1998) - mostra o número de empresa industriais em funcionamento por 1000 habitantes;

$\mathrm{X}_{7}$ - número de estabelecimentos comerciais por 1000 habitantes (1998);

$\mathrm{X}_{8}$ - número de empresas prestadoras de serviços por 1000 habitantes (1998);

$\mathrm{X}_{9}$ - valor per capita, arrecadado de ICMS (1998) - parcela do ICMS arrecadado, por habitante;

$\mathrm{X}_{10}$ - valor per capita, adicionado de ICMS (1998) - parcela do ICMS adicionado, por habitante. $\mathrm{O}$ valor adicionado é resultado da diferença entre a base de cálculo da compra de mercadorias e a base de cálculo da sua venda;

$\mathrm{X}_{11}$ - Produto Interno Bruto a preço de mercado, per capita (1998) - é o valor monetário dos bens e serviços finais produzidos por habitantes do município;

$\mathrm{X}_{12}$ - percentual de crianças de zero a 11 meses, só mamando (1998);

$\mathrm{X}_{13}$ - complementar de percentual de crianças de zero a 11 meses, subnutridas (1998);

$\mathrm{X}_{14}$ - complementar de percentual de crianças de 12 a 23 meses, subnutridas (1998);

$\mathrm{X}_{15}$ - participação da produção agrícola total do município na produção agrícola total do Estado (1995);

$\mathrm{X}_{16}$ - inverso da média da salinização vezes 1000 , medida por mg/litro;

$\mathrm{X}_{17}$ - inverso da média da salinidade ponderada pelo número de famílias atendidas; 
$\mathrm{X}_{18}$ - complementar do percentual de poços com teor de sal acima 1000mg/litro de STD;

$\mathrm{X}_{19}$ - participação (\%) da produção vegetal do município sobre o total da produção vegetal do Estado (1995) e

$\mathrm{X}_{20}$ - participação (\%) da produção animal do município sobre o total da produção animal do Estado (1995);

\subsection{Análise Fatorial}

A análise simultânea de um conjunto que contempla inúmeras variáveis correlacionadas exige a aplicação de uma metodologia capaz de simplificar um grande vetor de dados em um conjunto reduzido de variáveis. A aplicação da análise fatorial permite essa simplificação, reduzindo esse grande vetor de dados correlacionados a um conjunto menor de variáveis não observáveis, denominadas fatores ortogonais, captando, entretanto, o máximo possível da variância das variáveis que lhes deram origem.

Segundo Johnson e Wichern (1978), quando as variáveis explicativas de uma equação a ser ajustada apresentam significativo grau de intercorrelação, ou quando o objetivo é efetuar a classificação por intermédio da criação de um índice, o método mais indicado para a extração dos fatores é o dos componentes principais.

Como o objetivo deste trabalho é a construção de um índice, o instrumental de análise utilizado será, então, análise fatorial pelo método dos componentes principais.

A aplicação da análise fatorial pode ser resumida em quatro etapas. A primeira consiste na determinação da matriz de correlação ou da matriz de variância-covariância das variáveis originais. Quando estas são medidas em escala e unidades muito diferentes entre si, a análise fatorial deve ter como ponto de partida a matriz de correlação entre as variáveis. Não sendo esse o caso, pode-se, alternativamente, partir da matriz de variância-covariância. Em qualquer caso, o método de extração dos fatores é o dos componentes principais; adota-se como fatores comuns os " $m$ " primeiros componentes principais.

Antes de extrair esses fatores, faz-se necessário verificar, a partir da matriz de correlação ou da matriz de variância-covariância, a 
adequabilidade do conjunto de variáveis ao procedimento estatístico, a partir da análise da estrutura de interdependência das variáveis. A análise fatorial exige que as variáveis apresentem determinado grau de correlação.

Assim, freqüentemente, a seleção das variáveis é feita, utilizando-se a medida de adequação da amostra MSA (measure of sampling adequacy), a qual é obtida da seguinte forma:

$$
\operatorname{MSA}_{\mathrm{i}}=\sum \mathrm{r}_{\mathrm{ij}}^{2} \div\left(\sum \mathrm{r}_{\mathrm{ij}}^{2}+\sum \mathrm{a}_{\mathrm{ij}}^{2}\right), \text { com } \mathrm{i} \neq \mathrm{j}
$$

onde $\mathrm{r}_{\mathrm{ij}}$ e $\mathrm{a}_{\mathrm{ij}}$ são, respectivamente, o coeficiente de correlação simples e o coeficiente parcial de correlação entre as variáveis i e j.

A consistência geral dos dados pode ser aferida pelo método de Kayser Mayer Olkim, calculando-se o índice conhecido como KMO. Por esse método, compara-se a magnitude dos coeficientes de correlação observados com os coeficientes de correlação parcial, obtendo-se o índice KMO.

O resultado será um número entre zero e um, sendo tanto melhor o índice quanto mais próximo de um.

Outro teste importante, pois reforça o resultado obtido com o KMO, é o chamado teste de esfericidade de Bartlett. Por meio deste, pode-se saber se a correlação entre as variáveis é significativa e se a variabilidade dos dados pode ser representada por um número pequeno de fatores. A aplicação da análise fatorial será adequada se o nível de significância for pequeno.

Para se proceder à análise fatorial a partir da matriz de correlação, faz-se necessário efetuar a normalização das variáveis. Isto significa centrá-las em torno da média aritmética e medi-las em termos de unidades de desvio-padrão.

Assim, seja $\mathrm{z}_{\mathrm{i}}$ uma variável normalizada ou padronizada, então:

$$
\mathrm{Z}_{\mathrm{i}}=\left(\mathrm{X}_{\mathrm{i}}-\mu\right) / \sigma_{\mathrm{i}}=\mathrm{x}_{\mathrm{i}} / \sigma_{\mathrm{i}}
$$

Na qual $\sigma_{\mathrm{i}}$ é o desvio padrão da variável $\mathrm{X}_{\mathrm{i}, \mathrm{e}} \mu$ a média aritmética. Concluídos esses procedimentos preliminares, a segunda etapa consiste na extração dos fatores que representarão o conjunto de dados. 
Não há critérios definidos quanto ao número mais adequado de fatores que devem representar determinado conjunto de variáveis. Os fatores, todavia, devem ser em número suficientemente reduzido, mas que, em conjunto, captem a maior percentagem possível das variâncias das variáveis selecionadas. ${ }^{5}$

Nessa etapa são definidas as cargas fatoriais e comunalidades ${ }^{6}$. A correlação de cada variável com os fatores é expressa, em termos algébricos, por:

$$
\mathrm{X}_{\mathrm{i}}=\mathrm{a}_{\mathrm{i} 1} \mathrm{~F} 1+\mathrm{a}_{\mathrm{i} 2} \mathrm{~F} 2+\ldots+\mathrm{a}_{\mathrm{in}} \mathrm{Fn}+e
$$

Cada variável observada $\left(\mathrm{X}_{1}, \mathrm{X}_{2}, \mathrm{X}_{\mathrm{n}}\right)$ é expressa como combinação linear dos fatores, mais um termo residual $(e)$ que representa a parte não explicada pelos fatores. Os fatores (Fi), por sua vez, são combinados por meio das cargas fatoriais, representadas pelas constantes "a".

A terceira etapa da análise fatorial consiste na transformação ortogonal, ou simplesmente rotação, da matriz das cargas fatoriais ${ }^{7}$, que pode ser feita por diferentes métodos.

A quarta etapa da análise fatorial consiste na determinação da matriz dos escores fatoriais. Esta é obtida a partir da seguinte fórmula:

$$
\mathrm{EF}=\mathrm{A}^{\mathrm{t}} \cdot \mathrm{R}^{-1} \cdot \mathrm{X}^{\mathrm{t}}
$$

\footnotetext{
${ }^{5}$ Alguns autores como Lawley (1940) e Kaiser (1960), entretanto, propõem critérios estatísticos para determinar a quantidade ideal de fatores. Para Kaiser (1960), só se deve extrair aqueles fatores que apresentarem raízes características (eigenvalues) superiores à unidade. As raízes características são resultados da soma do quadrado das cargas fatoriais dos fatores e refletem a importância relativa de cada fator. A razão entre uma raiz característica e a soma das variâncias (ou o número das variáveis, na matriz de correlação) mostra a proporção da variância total do modelo que é explicada pelo fator.

${ }^{6}$ As cargas fatoriais expressam as covariâncias entre cada fator e as respectivas variáveis originais. No caso da matriz de correlação (variáveis padronizadas), as cargas fatoriais correspondem às correlações entre os fatores e as variáveis originais. A comunalidade é obtida pelo somatório das cargas fatoriais ao quadrado em cada um dos fatores utilizados. Esta expressa, por meio de um índice, o quanto da variância de cada variável é explicado pelo modelo. Desse modo, quanto mais próximas de um estiverem as comunalidades, melhor ajustado estará o modelo.

7 A rotação visa a deixar as variáveis, que compõem um determinado fator, mais fortemente correlacionadas entre si e com maior grau de independência em relação às variáveis que compõem os outros fatores. Com isso consegue-se dar melhor significado interpretativo para os fatores.
} 
onde EF é a matriz dos escores fatoriais; $\mathrm{A}^{\mathrm{t}}$, a transposta da matriz das cargas fatoriais; $\mathrm{R}^{-1}$, a matriz inversa da matriz de correlação; e $\mathrm{X}^{\mathrm{t}}$, a transposta da matriz dos dados originais padronizados.

Os escores fatoriais de cada fator possuem distribuição normal, com média zero e variância unitária e, desse modo, podem ser utilizados para indicar a posição relativa de cada observação relativamente ao conceito expresso pelo fator ${ }^{8}$. A partir da matriz dos escores fatoriais, é possível construir-se um índice para hierarquizar as observações, ponderando adequadamente os escores de cada fator.

Assim, a construção desses índices é feita a partir da matriz dos escores fatoriais e pode se dar pela soma, simples ou ponderada, dos escores, pertencentes a uma mesma observação.

Como os fatores captam em níveis diferentes as variâncias das variáveis, uns mais e outros menos, é razoável que, na construção de um índice envolvendo todos os fatores, seja utilizado critério de ponderação capaz de conferir a cada fator sua importância relativa. No caso, a raiz característica, por expressar a capacidade de cada fator em captar a variância das variáveis, se presta ao papel de termo de ponderação.

\subsection{Análise de agrupamento}

Obtidos os índices, pode-se, ainda, efetuar um procedimento conhecido como análise de agrupamento ou análise de cluster $^{9}$. Trata-se de um recurso metodológico que visa a agrupar em classes um conjunto de informações, de forma que os dados dos indivíduos no interior dos grupos formados sejam os mais similares possíveis e os grupos sejam os mais diferentes possíveis uns dos outros.

Neste trabalho utilizou-se a técnica de partição pelo algoritmo das K-médias.

\footnotetext{
${ }^{8}$ Quanto mais distante de zero, positivamente, for o escore fatorial daquela observação, melhor será a posição relativa daquela observação, naquele fator.

${ }^{9}$ Optou-se pela análise de cluster por esta ser um importante complemento de análise, uma vez que determinados atributos se tornam mais evidentes se avaliados a partir de grupos do que de indivíduos isoladamente.
} 
Assim, seja $\mathrm{P}(\mathrm{j})$ a j-ésima classe de agrupamento, tem-se que:

$\mathrm{p}(\mathrm{j})=\left\{\mathrm{o}_{\mathrm{i}}(\mathrm{j})\right\}$, com $\left.1 \leq \mathrm{i} \leq \mathrm{nj}\right\}$

onde

$\mathrm{p}(\mathrm{j})$ - classe de agrupamento $\mathrm{j}$;

$\mathrm{o}_{\mathrm{i}}(\mathrm{j})$ - coordenada $\mathrm{i}$, da classe $\mathrm{j}$; $\mathrm{e}$

O centro de cada classe de agrupamento será a média das coordenadas de seus elementos. A soma dos quadrados residuais dentro do $\mathrm{j}$ ésimo grupo SQRes(j) será dada por:

$\operatorname{SQRes}(\mathrm{j})=\sum \mathrm{d}^{2}\left[\mathrm{o}_{\mathrm{i}}(\mathrm{j}) ; \quad \overline{\mathrm{o}}(\mathrm{j})\right] \quad[1 \leq \mathrm{i} \leq \mathrm{nj}]$

Onde

$\overline{\mathrm{O}}(\mathrm{j})$ é a média das coordenadas dos elementos “ $\mathrm{j}$ ” e

$\mathrm{d}^{2}$ é o quadrado da distância euclidiana do elemento “i”, da classe “j”, ao seu centro;

Para a partição como um todo, a soma dos quadrados residuais será:

SQRes $=\sum \operatorname{SQRes}(\mathrm{j}), \operatorname{com}[1 \leq \mathrm{i} \leq \mathrm{nj}]$

Quanto menor for o valor de $\mathrm{d}^{2}$, menor será a SQRes(j) e, portanto, mais homogêneos serão os elementos dentro da classe e melhor será a partição processada.

\section{3 - Resultados e Discussão}

Com a aplicação da metodologia ${ }^{10}$ proposta obteve-se o índice KMO foi de 0,69 , revelando que o conjunto de variáveis se ajusta bem ao procedimento de análise fatorial. Em reforço, foi realizado o teste de Bartlett, tendo-se obtido os seguintes resultados: qui quadrado de 1.497,527; graus de liberdade de 190 e nível de significância $=0,000$.

${ }^{10}$ Para efetuar a análise fatorial, utilizou-se o programa de computador Statistical Package (SPSS 10.0) 
Todas as variáveis envolvidas na análise apresentaram comunalidade superior a 0,5 , mostrando que mais da metade da variância das variáveis é reproduzida pelos fatores ${ }^{11}$.

Foram selecionados sete fatores com raízes características superiores à unidade, que em conjunto, explicam $72,22 \%$ das variâncias das variáveis selecionadas, conforme tabela 1.

Visando a uma melhor definição dos conceitos dos fatores obtidos, processou-se a sua rotação ortogonal pelo método VARIMAX ${ }^{12}$, o qual apresentou melhores resultados, isto é, melhor definiu a correlação entre os fatores e as variáveis.

A matriz das cargas fatoriais mostra o grau de correlação das variáveis com cada fator. Vale lembrar que o conceito de cada fator é definido pelas variáveis que se apresentam mais fortemente correlacionadas com esse fator e, a partir da matriz das cargas fatoriais, se construiu a Tabela 1.

${ }^{11}$ Vale lembrar que a aplicação da análise fatorial possibilita a redução de grande vetor de dados correlacionados a um conjunto menor de variáveis não observáveis, denominadas fatores ortogonais, ou simplesmente fatores, captando, todavia, o máximo possível da variância das variáveis que lhes deram origem.

${ }^{12}$ Para mais informações, ver Kaiser (1958) The Varimax Criterion for Analytic Rotation in Factor Analysis. Psychometrika, 23:187-200. 
Tabela 1: Fatores utilizados, variáveis explicativas de cada fator, raiz característica associada a cada fator; variância explicada pelo fator; variância acumulada e conceito expresso pelo fator

\begin{tabular}{|c|c|c|c|c|c|}
\hline Fator & $\begin{array}{l}\text { Indicadores } \\
\text { que definem } \\
\text { os fatores }\end{array}$ & $\begin{array}{c}\text { Raiz } \\
\text { Características }\end{array}$ & $\begin{array}{c}\% \text { da } \\
\text { variância } \\
\text { Explicada }\end{array}$ & $\begin{array}{l}\text { Variância } \\
\text { Acumulada }\end{array}$ & $\begin{array}{l}\text { Conceito } \\
\text { Expresso } \\
\text { pelo Fator }\end{array}$ \\
\hline 1 & $\mathrm{X}_{11}, \mathrm{X}_{10}, \mathrm{X}_{6}, \mathrm{X}_{8}$ & 2,658925 & 13,29463 & 13,29463 & $\begin{array}{l}\text { Potencial } \\
\text { econômico }\end{array}$ \\
\hline 2 & $\mathrm{X}_{16}, \mathrm{X}_{17}$ e $\mathrm{X}_{18}$ & 2,643354 & 13,21677 & 26,5114 & $\begin{array}{l}\text { Potabilidade da } \\
\text { água, definida } \\
\text { pelo teor de sal }\end{array}$ \\
\hline 3 & $\mathrm{X}_{15}, \mathrm{X}_{20}$ e $\mathrm{X}_{19}$ & 2,571212 & 12,85606 & 39,36746 & $\begin{array}{l}\text { Nível de } \\
\text { produção } \\
\text { agropecuária }\end{array}$ \\
\hline 4 & $\mathrm{X}_{13}, \mathrm{X}_{14}, \mathrm{X}_{12}$ & 1,937965 & 9,689826 & 49,05728 & Nutrição infantil \\
\hline 5 & $\mathrm{X}_{4} \mathrm{e} \mathrm{X}_{5}$ & 1,75507 & 8,775349 & 57,83263 & $\begin{array}{l}\text { Saúde, expressa } \\
\text { pela disponibili- } \\
\text { dade de profissi- } \\
\text { onais da saúde }\end{array}$ \\
\hline 6 & $\mathrm{x}_{3} \mathrm{e} \mathrm{x}_{2}$ & 1,462833 & 7,314167 & 65,1468 & Escolaridade \\
\hline 7 & $\mathrm{x}_{7}$ & 1,41527 & 7,076349 & 72,22315 & $\begin{array}{l}\text { Nível de atividade } \\
\text { comercial }\end{array}$ \\
\hline
\end{tabular}

Fonte: Dados da pesquisa

Em seguida, obteve-se a matriz dos escores fatoriais a partir da qual foram construídos os índices que serviriam de base para a hierarquização dos municípios.

Para melhor visualização dos resultados, os 170 municípios foram agrupados em três classes. Para tanto, processou-se uma análise de agrupamento pelo método das k-médias, descrito na parte metodológica. Os resultados dessa análise, associados a dados da pesquisa, foram sintetizados na Tabela 2. Eles revelam que a proporção do número de poços é quase equivalente ao da população, considerando que a primeira classe detém $22,36 \%$ da população e $25,06 \%$ dos poços; a segunda classe, $48,99 \%$ da população e $47,97 \%$ dos poços, e a terceira classe, $28,63 \%$ da população e $26,96 \%$ dos poços. Ressalte-se que os municípios pertencentes à primeira classe são os que apresentaram os piores índices, os da segunda classe são aqueles que apresentaram índices intermediários e os da terceira classe, os melhores índices. 
Outra importante informação que se pode extrair dessa tabela diz respeito à incidência do alto teor de sal (acima de 1000mg/litro de STD) nos poços perfurados nos municípios pertencentes a cada classe. É de se notar que os municípios agrupados na primeira classe, aqueles com piores índices, são os que apresentam maior percentual de poços com teor de sal acima de $1000 \mathrm{mg} /$ litro de STD (62,20\%). Nos municípios da terceira classe, porém, essa incidência é bem menor $(25,57 \%)$. Os municípios da faixa intermediária apresentam também proporção de incidência de sal numa faixa intermediária $(42,8 \%)$.

Tabela 2 - Número de municípios, percentual da população, percentual dos poços e percentual de poços com elevado teor de sal, segundo as classes dos municípios.

\begin{tabular}{|c|c|c|c|c|}
\hline Classes & $\begin{array}{c}\text { Número de } \\
\text { Municípios }\end{array}$ & $\begin{array}{c}\text { População } \\
(\%)\end{array}$ & $\begin{array}{c}\text { Participação } \\
(\%) \text { do Total }\end{array}$ & $\begin{array}{c}\text { Poços > 1000mg/l } \div \text { Total } \\
\text { de Poços }\end{array}$ \\
\hline 1 & 65 & 22,369771 & 25,062756 & 0,62203479 \\
\hline 2 & 83 & 48,996287 & 47,971991 & 0,42798127 \\
\hline 3 & 22 & 28,633942 & 26,965253 & 0,25575698 \\
\hline & 170 & 100,00 & 100,00 & \\
\hline
\end{tabular}

Fonte: Dados da pesquisa

Essas diferenças tendem a se tornar mais pronunciadas ao se observar os dados dos municípios classificados em posições mais distantes. Assim, por exemplo, quando se compara a incidência de sal nos poços dos dez primeiros municípios do ranking com a dos dez últimos, observa-se que os dez primeiros, juntos, atingem o percentual de $68,43 \%$; isto é, $68,43 \%$ do total de seus poços apresentam alto teor de sal, enquanto nos dez últimos esse percentual é de apenas $16,88 \%$.

Esses resultados corroboram a hipótese inicial deste trabalho de que existe forte correlação entre os indicadores socioeconômicos dos municípios e ocorrência de elevados níveis de salinização das águas que abastecem as populações desses municípios.

Os números da terceira e sétima colunas (ranking), da Tabela 3 variam de 01 a 170 e indicam a posição relativa de cada município. Assim, o município com classificação de número 01, no caso Ararendá, é aque- 


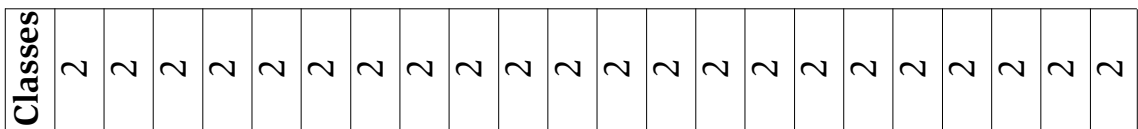

.

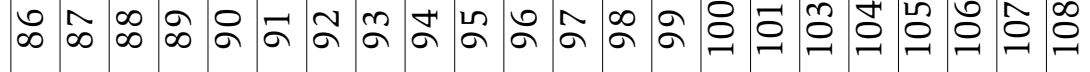
2

$\stackrel{2}{\sim} \infty$ in $n$ in テ

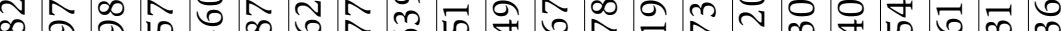

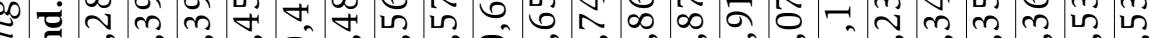

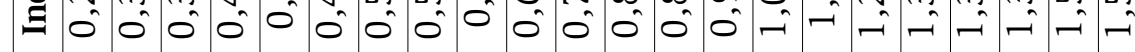

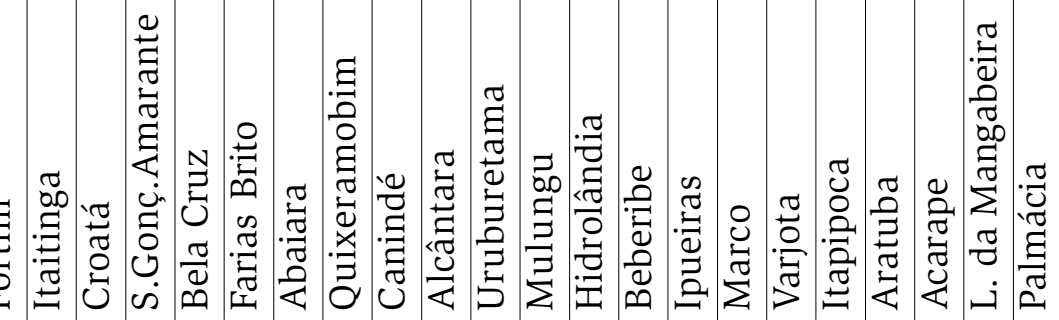

is

:

i⿱宀 을

ชึ

.

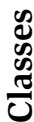

\&

$\infty$

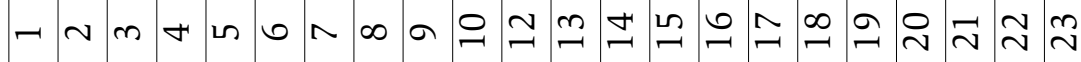

\section{西}

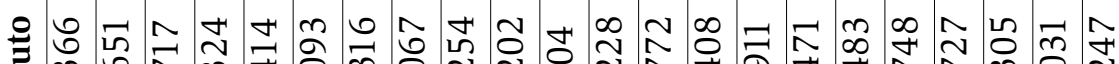

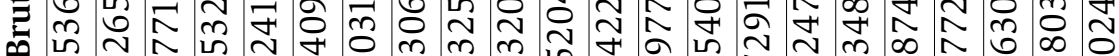

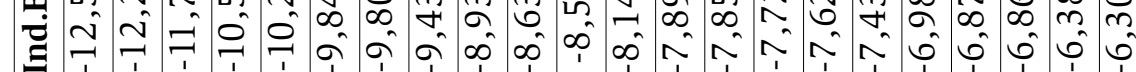

苞

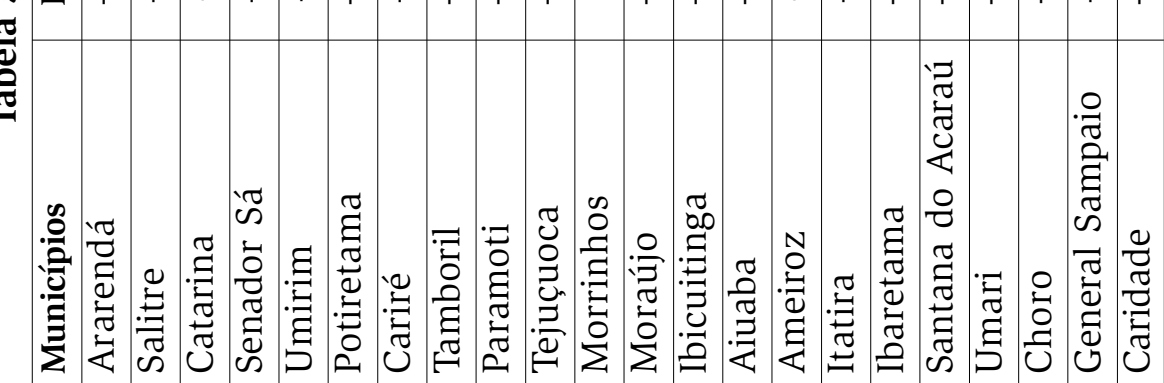


380 - Critério para Implantação de Tecnologias de Suprimentos de Água Potável em Municípios Cearenses Afetados pelo Alto Teor de Sal

\begin{tabular}{|c|c|c|c|c|c|c|c|c|c|c|c|c|c|c|c|c|c|c|c|c|c|c|c|}
\hline $\begin{array}{l}\mathscr{\infty} \\
\underset{\infty}{0} \\
\underset{0}{0}\end{array}$ & $\sim$ & $N$ & $\sim$ & $\sim$ & $\sim$ & $\sim$ & $\sim$ & $\sim$ & $\sim$ & $\sim$ & $\sim$ & $\sim$ & $\sim$ & $\sim$ & $\sim$ & $\sim$ & $\sim$ & $\sim$ & $\sim$ & $\sim$ & $\sim$ & $\sim$ & $\sim$ \\
\hline 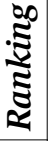 & ఠ口 & $\stackrel{\varrho}{\varrho}$ & 二 & $\underset{\sim}{\sim}$ & $\begin{array}{l}n \\
=1\end{array}$ & $\underset{\sigma}{\sqsupset}$ & 드 & $\underset{\sigma}{\sigma}$ & $\approx$ & $\infty$ & 可 & 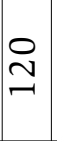 & $\widetilde{\sim}$ & $\underset{N}{N}$ & $\stackrel{n}{\sim}$ & $\underset{\sim}{\sim}$ & ปn & $\underset{\sim}{\sim}$ & $\widetilde{\sim}$ & $\stackrel{\infty}{\sim}$ & a & 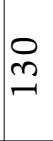 & $\vec{n}$ \\
\hline 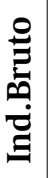 & $\begin{array}{l}\infty \\
\infty \\
0 \\
0 \\
0 \\
1 \\
-1\end{array}$ & 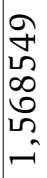 & $\begin{array}{l}\infty \\
\text { Un } \\
\sim \\
\infty \\
\infty \\
-1\end{array}$ & $\begin{array}{l}\text { న } \\
\infty \\
\check{\sigma} \\
\check{-}\end{array}$ & 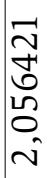 & 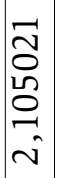 & $\begin{array}{l}\underset{\sim}{\sim} \\
\sim \\
\sim \\
v\end{array}$ & \begin{tabular}{l}
$\infty$ \\
+ \\
$\infty$ \\
\multirow{+}{+}{} \\
v \\
$\sim$ \\
$\sim$ \\
$\sim$
\end{tabular} & $\begin{array}{l}\tilde{n} \\
\tilde{\sigma} \\
\tilde{n} \\
\hat{v}\end{array}$ & $\begin{array}{l}+ \\
n \\
\infty \\
ن \\
0 \\
0 \\
\sim \\
\text { v }\end{array}$ & $\begin{array}{l}\sim \\
\infty \\
\infty \\
\sim \\
\sigma \\
\sim \\
\sim\end{array}$ & $\begin{array}{l}+ \\
ن \\
0 \\
n \\
\tilde{n} \\
\sim \\
\sim\end{array}$ & 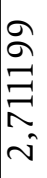 & $\begin{array}{l}\infty \\
\sigma \\
\sigma \\
0 \\
\infty \\
\sim \\
\sim\end{array}$ & 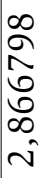 & \begin{tabular}{l}
0 \\
Un \\
\multirow{2}{*}{} \\
$\sigma$ \\
N
\end{tabular} & $\begin{array}{l}n \\
m \\
\tilde{n} \\
\tilde{n} \\
n \\
n\end{array}$ & $\begin{array}{l}n \\
\infty \\
0 \\
\tilde{\Omega} \\
\sigma \\
0 \\
n\end{array}$ & $\begin{array}{l}+ \\
\infty \\
\infty \\
\infty \\
\infty \\
m \\
m\end{array}$ & $\begin{array}{l}\tilde{D} \\
n \\
v \\
\dot{y} \\
m \\
m\end{array}$ & 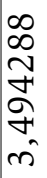 & $\begin{array}{l}0 \\
\text { 10 } \\
\infty \\
\infty \\
\text { 1n } \\
n\end{array}$ & $\begin{array}{l}a \\
m \\
m \\
\tilde{n} \\
\tilde{m} \\
m\end{array}$ \\
\hline 哭 & 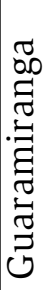 & 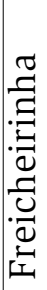 & 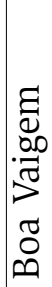 & 苟 & 里 & 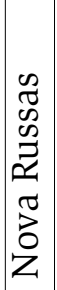 & 窇 & 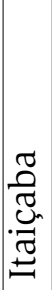 & 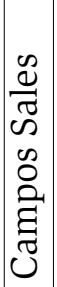 & 㖧 & 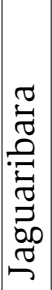 & 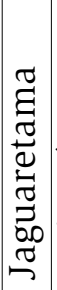 & 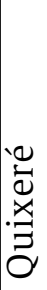 & 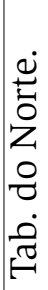 & 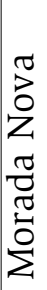 & 悉 & 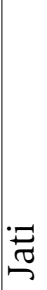 & 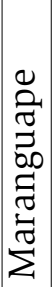 & 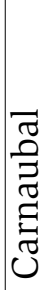 & 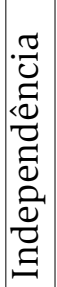 & 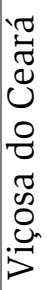 & 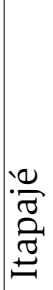 & 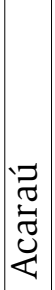 \\
\hline
\end{tabular}

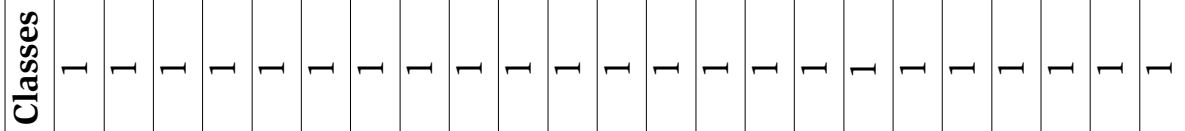

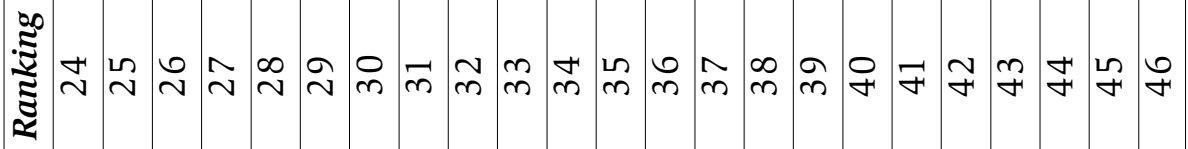

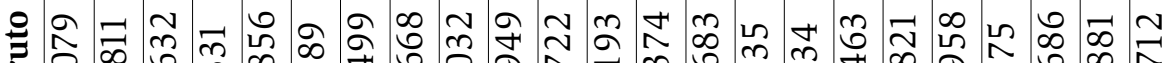
• E

\begin{tabular}{|c|c|c|c|c|c|c|c|c|c|c|c|c|c|c|c|c|c|c|c|c|c|c|c|}
\hline . & 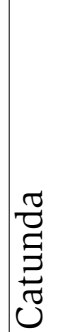 & 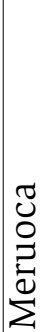 & 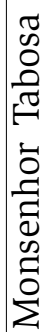 & 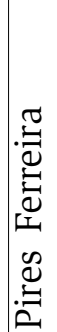 & 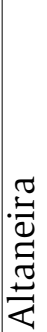 & 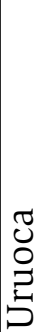 & 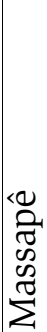 & حُ & 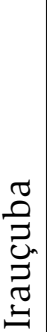 & $\begin{array}{l}0 \\
\underline{0} \\
0 \\
0 \\
0 \\
0 \\
0 \\
0 \\
0 \\
Z \\
Z\end{array}$ & 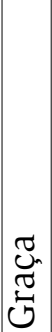 & 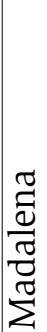 & 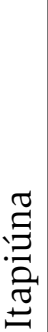 & 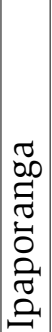 & 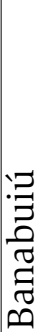 & 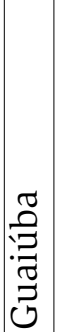 & 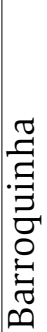 & 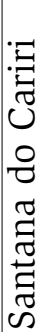 & 洗 & 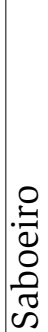 & 己 & 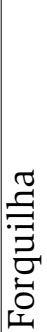 & 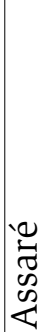 \\
\hline
\end{tabular}




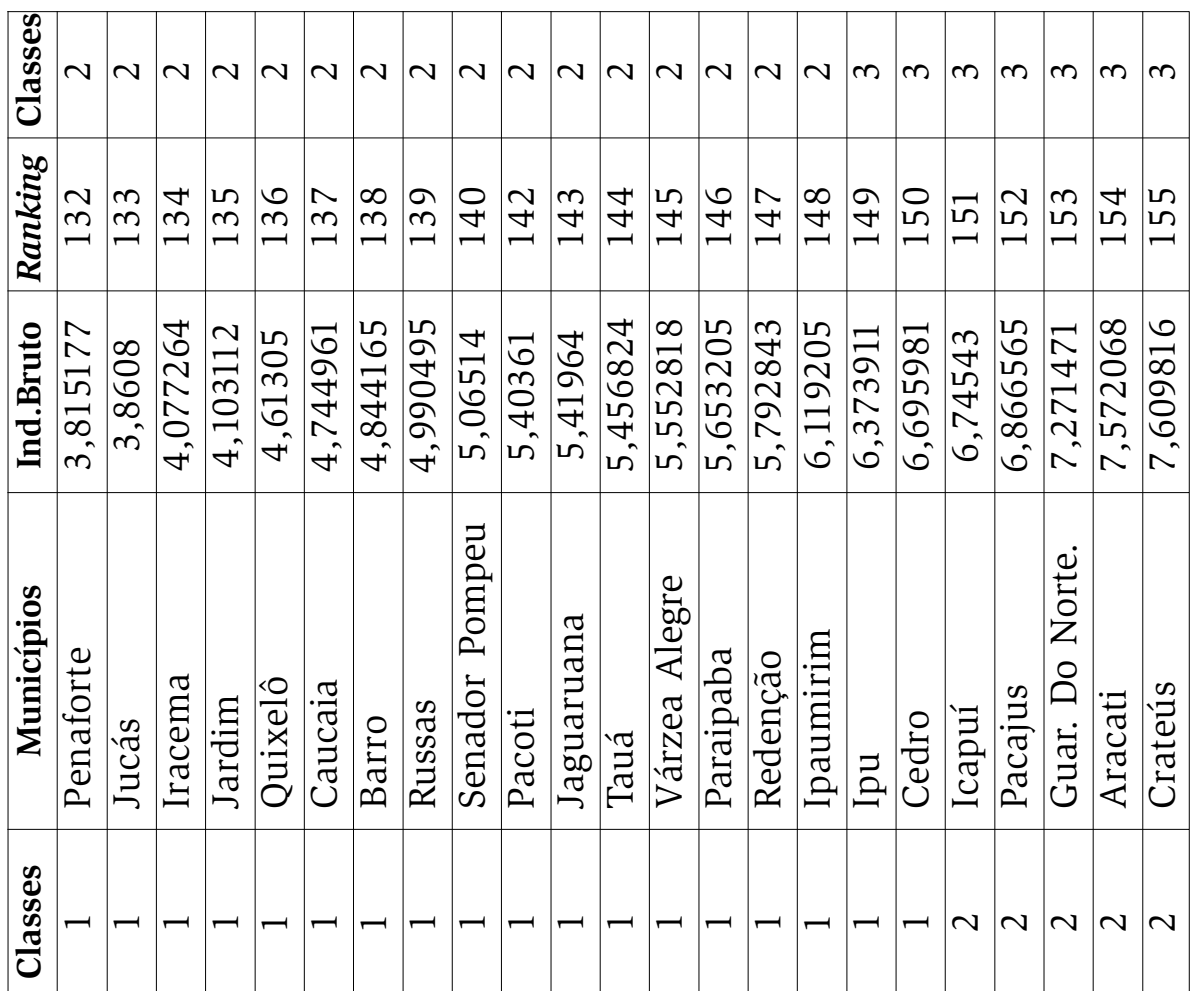

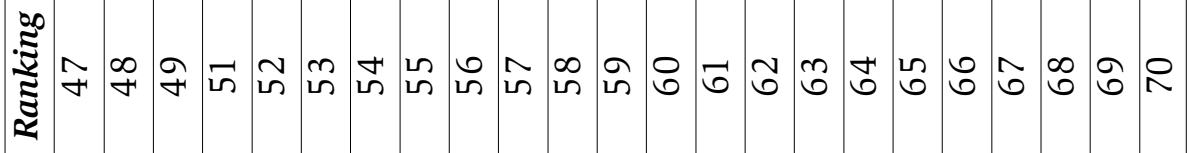

ํㅏㅂ

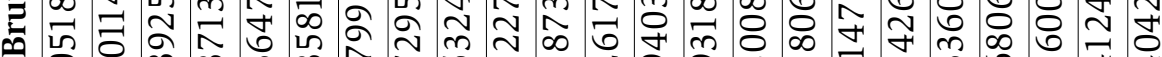

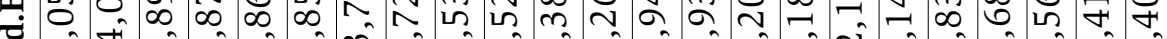

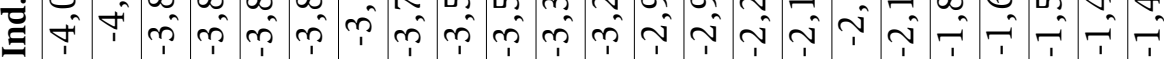

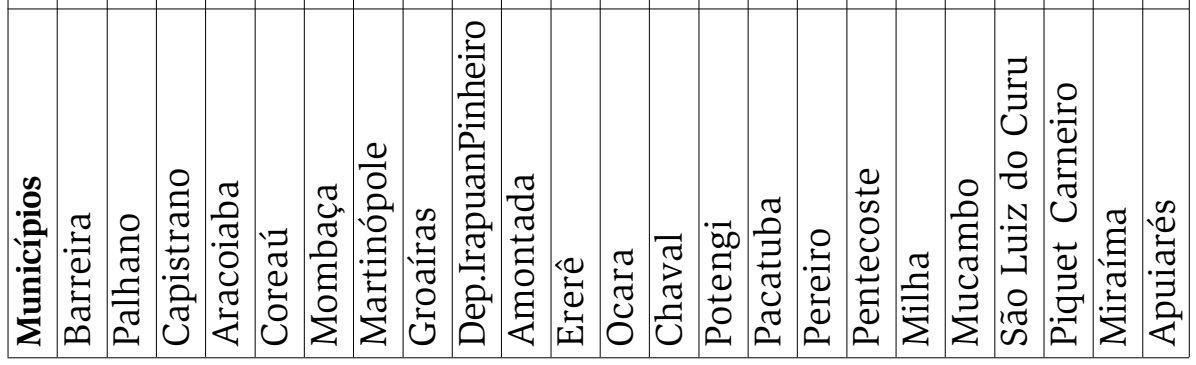


382 - Critério para Implantação de Tecnologias de Suprimentos de Água Potável em Municípios Cearenses Afetados pelo Alto Teor de Sal

\begin{tabular}{|c|c|c|c|c|c|c|c|c|c|c|c|c|c|c|c|c|}
\hline 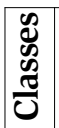 & $m$ & $m$ & $m$ & & $n$ o & $n$ & $n$ & $m$ & $m$ & $n$ & $m$ & $m$ & $m$ & $n$ & $m$ & $m$ \\
\hline 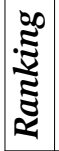 & 吕 & ํㅡㄴ & in & in & 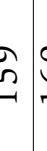 & 임 & 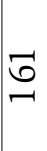 & 금 & 0 & ठ্ & ᄂ2 & ڤ્ర & $\widehat{\sigma}$ & $\infty$ & a & 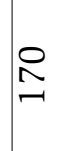 \\
\hline 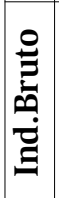 & \begin{tabular}{l}
$\infty$ \\
\\
2 \\
\multirow{J}{0}{} \\
$\infty$ \\
$\sim$ \\
$\sim$
\end{tabular} & 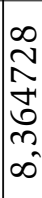 & $\begin{array}{l}n \\
\sigma \\
\sigma \\
\sigma \\
\infty\end{array}$ & $\left\{\begin{array}{l}7 \\
\widetilde{\sigma} \\
\tilde{J} \\
0 \\
\sigma\end{array}\right.$ & 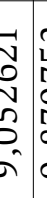 & $\begin{array}{l}N \\
\\
\\
\infty \\
\sigma\end{array}$ & $\begin{array}{l}n \\
\sigma \\
\infty \\
1 \\
0 \\
0 \\
-1\end{array}$ & 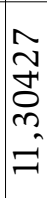 & 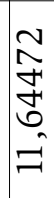 & \begin{tabular}{l}
$n$ \\
\multirow{4}{*}{} \\
2 \\
$\infty$ \\
$\infty$ \\
$=1$
\end{tabular} & 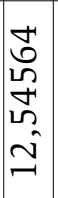 & 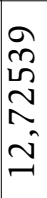 & 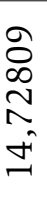 & 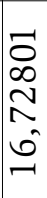 & \begin{tabular}{l}
$\infty$ \\
0 \\
\multirow{+}{*}{} \\
$\infty$ \\
$\infty$ \\
0 \\
-1
\end{tabular} & 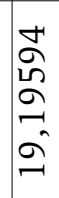 \\
\hline 总 & 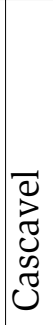 & 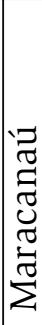 & 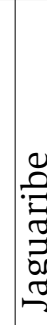 & 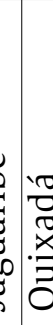 & 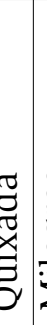 & 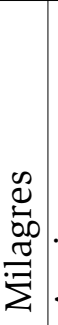 & 恣 & 击 & 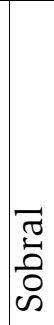 & 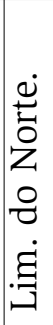 & 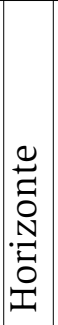 & 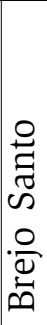 & 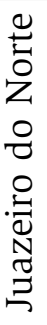 & م & 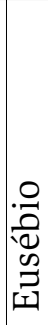 & 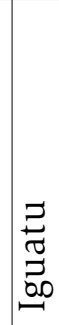 \\
\hline $\begin{array}{l}\mathscr{D} \\
\mathscr{D} \\
\underset{\Xi}{0}\end{array}$ & $v$ & $\sim$ & $\sim$ & & $\begin{array}{lll}\mathrm{N} & \mathrm{c}\end{array}$ & $\sim$ & $\sim$ & $\sim$ & $\sim$ & $\sim$ & $\sim$ & $\sim$ & $\sim$ & $\sim$ & $\sim$ & $\sim$ \\
\hline 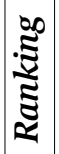 & $\nabla$ & $\stackrel{N}{N}$ & 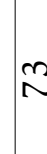 & 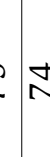 & + & $\stackrel{2}{\sim}$ & $\mathscr{n}$ & 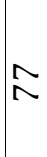 & $\stackrel{\infty}{n}$ & $\curvearrowright$ & $\infty$ & $\infty$ & $\infty$ & $\infty$ & $\underset{\infty}{+}$ & 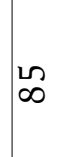 \\
\hline
\end{tabular}

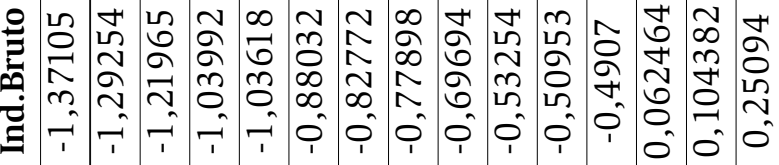

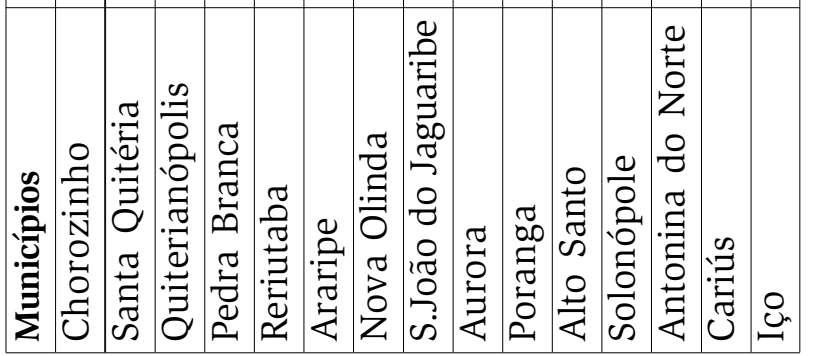


le que apresenta os piores índices e, portanto, deveria ser considerado prioritário para a instalação de equipamentos de dessalinização ou quaisquer outras tecnologias que solucionem o problema de suprimento de água potável. Na posição de número 02, está o Município de Salitre e assim sucessivamente.

Cumpre-se, portanto, dessa forma, a proposta inicial do trabalho de apresentar um critério para a alocação de tecnologia de abastecimento de água potável em municípios do Estado do Ceará a partir de uma hierarquização desses municípios, de acordo com os indicadores socioeconômicos e ambientais, inclusive os níveis de ocorrência de alto teor de sal na água subterrânea desses municípios.

É importante lembrar que, para a hierarquização dos municípios, não se levou em consideração a existência de fontes alternativas de abastecimento. De acordo com a hipótese deste trabalho, entretanto, nesses casos, como os poços com água salgada não abastecem a população, o resultado coerente seria esses municípios não serem classificados como prioritários para a instalação de equipamentos de dessalinização. Isto significa que a maior abrangência e interação das informações imprimem maior precisão ao critério proposto, fundamentado no modelo multivariado.

São emblemáticos os casos dos Municípios de Sobral e Limoeiro do Norte, os quais, embora apresentando percentual elevado de poços com alto teor de salinização, foram classificados, respectivamente, nas posições 163 e 164, portanto, figurando entre os últimos, na escala de prioridades (Tabela 4). Note-se, todavia, que ambos os municípios dispõem de outras opções, em condições satisfatórias, de abastecimento para as populações e, portanto, de fato, não deveriam ser prioritários. Estudos realizados confirmam que os municípios ora referidos, de fato, contam com boas fontes alternativas de abastecimento humano. Ver a esse respeito Pinheiro et al (2000) e Ceará (1994). 
Tabela 4 - Municípios com melhores índices, número total de poços, número de poços com teor de sal maior do que $1000 \mathrm{mg} /$ litro de STD e posição no ranking

\begin{tabular}{|l|c|c|c|c|c|}
\hline \multicolumn{1}{|c|}{ Municípios } & Classe & $\mathrm{N}^{0}$ Total Poços & $\mathrm{N}^{0}$ poços $>1000$ & \% poços > 1000 & Ranking \\
\hline Iguatu & 3 & 104 & 9 & 8,653846 & 170 \\
\hline Eusébio & 3 & 272 & 12 & 4,411765 & 169 \\
\hline Tianguá & 3 & 41 & 2 & 4,878049 & 168 \\
\hline Juazeiro do Norte & 3 & 64 & 2 & 3,125 & 167 \\
\hline Brejo Santo & 3 & 79 & 6 & 7,594937 & 166 \\
\hline Horizonte & 3 & 51 & 13 & 25,4902 & 165 \\
\hline Limoeiro do Norte & 3 & 59 & 28 & 47,45763 & 164 \\
\hline Sobral & 3 & 148 & 80 & 54,05405 & 163 \\
\hline Mauriti & 3 & 122 & 1 & 0,819672 & 162 \\
\hline Aquiraz & 3 & 250 & 31 & 12,4 & 161 \\
\hline
\end{tabular}

Fonte: Dados da pesquisa

O caso desses municípios reforça a proposta metodológica deste trabalho. Se a hierarquização fosse feita apenas com base nos dados de salinização dos poços, os Municípios de Sobral e Limoeiro do Norte seriam classificados como prioritários, em detrimento de outros, eventualmente com menor incidência de poços com água salinizada, porém sem dispor de fontes alternativas.

É importante, portanto, fazer a ressalva de que a metodologia proposta neste trabalho para a definição de prioridades na instalação de tecnologia de abastecimento de água potável não exclui a utilização de outros critérios coadjuvantes, ou que se lhe agreguem outras informações complementares para fins de tomada de decisão.

\section{4 - Conclusões}

Os resultados obtidos neste trabalho revelaram que o potencial econômico e a potabilidade das águas, medida pelo teor de sal, foram os fatores que mais contribuíram para a construção dos índices socioeconômicos dos municípios analisados.

Esses resultados mostram o quão importante é a potabilidade das águas na determinação das condições socioeconômicas das po- 
pulações dos municípios cearenses, legitimando a adoção desse índice como critério para alocação de equipamento de dessalinização ou opções tecnológicas outras voltadas para o suprimento de água potável.

Confirmando a hipótese inicial do trabalho, os resultados mostraram haver forte correlação entre os índices socioeconômicos e o teor de sal encontrados nos poços dos municípios. É interessante registrar que os municípios com baixos índices socioeconômicos apresentam índices elevados de salinização das águas, sendo que o inverso não é necessariamente verdadeiro. Isto é, municípios com alto teor de sal em suas águas subterrâneas podem eventualmente apresentar bons índices socioeconômicos. São os casos de Sobral e Limoeiro do Norte que, dentre outros fatores, possuem boas fontes de suprimento de água potável.

Por tudo isso, fica evidenciado que a construção de índices dos municípios a partir da análise fatorial com base em indicadores socioeconômicos da população representa critério tecnicamente consistente para estabelecer prioridades na instalação de dessalinizadores ou tecnologias outras que viabilizem o abastecimento de água potável para consumo humano.

\section{5 - Referências Bibliográficas}

BARROS, F.F. Estudos da qualidade da água e balanço de sais nos reservatórios superficiais na bacia do rio Curu. 128p.Dissertação (mestrado em Recursos Hídricos) Universidade Federal do Ceará, Fortaleza,1994

CEARÁ Secretaria de Recursos Hídricos. Subsídios para implantação do Plano Plurianual-1992-1995, Fortaleza, 1994. 78p.

CEARÁ Secretaria de Recursos Hídricos. Estudos de salinização dos reservatórios de água no Estado do Ceará. Fortaleza, 1996. 83p.

COMPANHIA DE PESQUISA DE RECURSOS MINERAIS (CPRM) 
Atlas dos Recursos Hídricos Subterrâneos do Ceará, Fortaleza, 2000.

Fundação Instituto de Pesquisa e Informação do Ceará (IPLANCE) Anuário Estatístico do Ceará 1998. Fortaleza, 2000. V.7, 2 Tomos.

JOHNSON, R.A.; WICHERN, D.W Applied regression analysis and other multivariate, New Jersey: Prentice Hall. 1978, 357p.

KAISER, H.F The varimax criterion for analytic rotation in factor analysis. Psychometrika, Massachussetts, v23, n 1:187200.Jul.1958.

KAISER, H.F. The aplication of eletronic computers to factor analysis. Educational and Psychological Mensurement, v.20.p.111-117.1960

LAWLEY, D.N. The estimation of factor loandings by the method of maximum likelihood. Proceedings of the Royal Society of Edinburgh, v,.60, n 2,.p. 47-58.abr 1940.

MATSUI, E. Origem e dinâmica da salinização de água do Nordeste brasileiro. Bacia do Rio Pajeú - PE, 1978, 164p. Tese de doutoramento - Escola Superior de Agricultura Luiz de Queiroz, Universidade de São Paulo, Piracicaba, 1978. 132p.

PINHEIRO, J.C.V. et al. Estudos do potencial agrícola do Rio Acaraú, Relatório Técnico Pro-Reitoria de Extensão-UFC/ SEBRAE. 2000

REBOUÇAS, A . C. Le problème de l"eau dans la zone semi-aride du Brêsil. 1973 159p.Tese (Doc., ès Sci) - Universidade de Strasbourg, Strasbourg, 1973

SANTIAGo, M. M. F. Mecanismo de salinização em regiões semiáridas; estudo dos açudes Pereira de Miranda e Caxitoré no Ceará 
1984. 176p. Tese (Tese de doutoramento em Geologia Geral e de Aplicação) Universidade de São Paulo, São Paulo. 1984.

Recebido em novembro de 2003 e revisto em março de 2004. 